\title{
Optimal Finite Element Modelling and 3-D Parametric Analysis of Strong Coupled Resonant Coils for Bidirectional Wireless Power Transfer
}

\author{
Babatunde Olukotun \\ Mechanical Engineering \\ Department, \\ University College London \\ London, United Kingdom \\ ucembol@ucl.ac.uk
}

\author{
J.S. Partridge \\ Mechanical Engineering \\ Department, \\ University College London \\ London, United Kingdom \\ julius.partridge09@ucl.ac.uk
}

\author{
Richard W. G. Bucknall \\ Mechanical Engineering \\ Department, \\ University College London \\ London, United Kingdom \\ r.bucknall@ucl.ac.uk
}

\begin{abstract}
Resonant coils can be utilised for the wireless transfer of power between a supply grid and an electric vehicle. In order to achieve an affordable and optimal resonant coil design, the coupling factor becomes a significant parameter. The coupling factor $(k)$ indicates the percentage of the total magnetic flux responsible for the transfer of electrical power from the transmitter to the receiver. A higher value of $k$ will increase the magnitude of real power transferred between coils as well as reduce in the proportion of reactive power circulating in the system. For optimal and efficient bidirectional Wireless Power Transfer (WPT) operation, the primary and secondary coils must be designed to achieve a high $Q$ uality factor $(Q)$ and high coupling factor. Using the same length of copper wire and volume of ferrite core, three simple resonant coils using finite element modelling (FEM) designs are proposed: circular, rectangular and doublesided winding coils. The physical length $(D)$ of the coils is restricted to be no more than $600 \mathrm{~mm}$ due to the physical diameter of most electric cars as well as regulations governing electromagnetic radiations. Magnetostatic performance analyses of the coil designs to confirm mutual inductances, coupling factors, the maximum magnetic flux density (B) distributions and maximum saturation current tolerances for the magnetic core over an airgap of $150 \mathrm{~mm}$ were carried out. Likewise, parametric performance evaluations of the different resonant coil designs in three-dimensional space comprising of airgap variation, longitudinal and lateral misalignment were undertaken. From the FEA and Parametric performance results, the model of an optimal design was evaluated through simulation and was found to achieve a strong magnetic coupling factor of more than 0.5 across an airgap of $170 \mathrm{~mm}$.
\end{abstract}

Keywords- Electric Vehicles, Bidirectional WPT, Vehicle-to-Grid, smart grids, resonant coils, magnetostatic analysis, coupling factor, quality factor, mutual inductance, magnetic flux density, finite element modelling.

\section{INTRODUCTION}

Wireless Power Transfer (WPT) Technologies can be traced back to the $20^{\text {th }}$ century through the invention of the Tesla Coil by Nikola Tesla, who wished to create a radial electromagnetic wave of about $8 \mathrm{~Hz}$ frequency, transmitted from the earth to the ionosphere, transferring electrical energy in the process[1]. WPT technology is a process of transferring electrical power from one point to another across space or vacuum without the use of any direct physical electrical conductor or medium. The technology can be divided into three categories: Electromagnetic Radiation-based WPT; Magnetic Resonant Coupled-based WPT; and Inductive coupled-based WPT[1].

The inductive-based WPT uses the principle of magnetic field induction using the near-field transmission technique. It is simple in design with high level of safety and mainly used in applications such as the charging of mobile phones, laptops, toothbrush and medical implants. Unfortunately, it has a major limitation in that its transmission distance is normally restricted to only a few centimetres[1]. Also it has limited tolerance to misalignment as there is a significant drop of power transfer efficiency (PTE) when the receiver coil is not optimally aligned with the transmitter coil. As a result, it is not suitable for charging of electric vehicles (EVs).

The Electromagnetic Radiation based WPT uses strong directional and energy concentrated waves from a microwave source, using a wave guide which is then emitted uniformly from a transmitting antenna. The receiving antenna then collects the microwave energy and converts it to electrical energy[1]. The electromagnetic radiation-based WPT has the advantage of transmission over a longer distance but there are safety issues related to radiation concerns. As a result, this technique is only used for low power transmission and this is not particularly applicable for EV charging applications since the requirement is for a significant amount of power to be transmitted.

The Magnetic Resonant coupling-based approach enables efficient power transfer between two electromagnetic subsystems operating at a resonant frequency[2]. In this approach, the transmitter coil is excited by an electrical source, which is then electromagnetically coupled with the receiver coil allowing power to be transferred[2]. This method of WPT has the advantage of longer distance power transmission compared with the inductive WPT technology. It also does not have the radiation concerns allied to the electromagnetic radiation-based 
WPT technology and offers better misalignment tolerance when compared with that of the inductive WPT technology whilst offering high power capability suitable for EV wireless charging application.

For maximum efficiency during power transfer, there are two important parameters: the Quality-factor of the resonators $(\mathrm{Q})$ and the mutual coupling factor $(\mathrm{k})$ between the two resonating coils[3]. The Q-factor is the ratio of the inductive reactance of the coil to its electrical resistance. The higher the Q-factor, the better the performance of the coil since the inductive properties become more pronounced and are responsible for the amount electrical power transferred between resonant coils [1]. The coupling factor $(\mathrm{k})$ is a measure of the ratio of the electromagnetic flux linkage between the receiver coil and the transmitter coil. Generally, electrical power transformers have very high coupling factors due to the presence of cores with no airgap between the primary and secondary coils. Coupled coils having $\mathrm{k}$ factors $\leq 0.5$ are termed as loosely or poor coupled coils whilst those coils having a $\mathrm{k}$ factor $\geq 0.5$ are called tightly or strong coupled coils[4]. An optimal coil design will need to achieve a $\mathrm{k}$ factor value of close to or greater than 0.5 as this provides for a reduction of reactive power circulating in the WPT. This in turn reduces the electrical components needed for the compensation of the leakage magnetic flux due to the coils being loosely coupled [4].

In order to reduce the material cost as well as the time in the design of optimal resonant coils, there is need to validate the design tool that will be used for the physical design modelling. Due to the difficulty involved in the analytical modelling of resonant coils, a finite element modelling (FEM) approach is employed for the design of complex physical coil designs. The procedure employs the use of approximation of the expected practical design parameters. Ansys FEM software is a popular tool used for such design and analysis and was employed for the proposed design.

To verify the accuracy of the proposed model design, the design environment, physical modelling and boundary condition were modelled after the design template of [5], [6] within $\pm 6 \%$ error.

\section{PROPOSED COIL SPECIFICATION AND DESIGN}

The electromagnetic resonance WPT technology is widely exploited for its high Power Transfer Efficiency in many electrical and electronic applications including the charging of electric vehicles. The technology is recognised for quick charging, optimal transmission of power across a frequency range and ease of control. The technology can be used for both dynamic and static charging of electric vehicles[7]. The electromagnetic resonance-based WPT technology normally employs the use of capacitors connected to both the secondary and primary coils, to compensate for the flux leakage as a result of increased airgap and the divergent nature of the electromagnetic flux. An LC circuit is used to enable efficient transfer of power at a particular resonant frequency.

According to [4], there are basically two types of magnetic resonance based WPT methods: the low frequency and high frequency magnetic resonance based WPT methods. The low frequency method normally operates at a resonant frequency lower than $200 \mathrm{kHz}$ while employing the use of a ferrite core to boost the magnetic coupling between the resonant coils. On the other hand, the high frequency method operates with frequency higher than $1 \mathrm{MHz}$ and without the need for a ferrite core.

The proposed optimal WPT technology makes use of the low frequency magnetic resonance coupling-based method for wireless charging of EVs. Designing an optimal resonant coil with high coupling factor involves using a given length of copper wire to create different shapes configuration wrapped around a ferrite core within a confined physical space and limit. The optimal design must have a good performance characteristics with respect to the airgap, lateral displacement, longitudinal displacement as well as angular displacement.

According to the work of [8], at constant Q-factor, if the coil diameter (D) is greater than twice the length of the airgap $(H)$, the PTE of the resonant coils will be greater than $80 \%$. With the coil diameter (D) greater than four times the length of the airgap, a PTE of close to $90 \%$ can be achieved. According to the work of [5], and with consideration to safety regulations, the range of airgap for most electric vehicles varies from $11 \mathrm{~cm}$ to $20 \mathrm{~cm}$. Using the maximum airgap of $20 \mathrm{~cm}$, in order to achieve a PTE between $80 \%$ and $90 \%$, a resonant coil of diameter ranging from $40 \mathrm{~cm}$ to $80 \mathrm{~cm}$ will be required. Considering safety requirements and the width of most EVs, an arbitrary diameter corresponding to the average length of both extreme values, which is $60 \mathrm{~cm}$, will be used. Also, Litz copper wire of length of $26.76 \mathrm{~m}$ will be used for the coil design in order to correctly model the different coil shapes within the $60 \mathrm{~cm}$ size limit.

Starting with three simple coil designs comprising circular, rectangular and double-sided winding coils with rectangular ferrite core using a rectangular aluminium shield sheet have been modelled. The physical design parameters of the three designs are illustrated in Table 1.

Based on the parameter specification of each of the resonant coil designs, a model for each design was built in Ansys Electronic Desktop environment..

\section{PARAMETRIC ANALYSIS OF THE RESONANT COILS}

Prior to the optimization of the existing simple designs, there is need to perform a core saturation analysis of the ferrite core used in order to determine the maximum current which the resonant coils may be subjected to, without the core attaining its saturation limit. In addition, a parametric sweep analysis of each coil design based on the variation of the airgap, lateral misalignment and longitudinal misalignment will be implemented.

The secondary coils' 3-dimensional positional measurement parameters are varied along the $\mathrm{x}, \mathrm{y}$ and $\mathrm{z}$ coordinate systems. The parametric sweep along the $\mathrm{x}$-axis corresponds to the lateral misalignment (green line), the parametric sweep along the y-axis corresponds to the longitudinal misalignment (the blue line), while the airgap variation is done through a parametric sweep along the z-axis (red line) in Figures 1(c), (d) and (e). 
TABLE I. TABLE of PARAMETER SPECIFICATIONS For Simple CoIl DESIGNS

\begin{tabular}{|c|c|c|c|}
\hline Parameter & $\begin{array}{c}\text { Circular Coil } \\
\text { Design }\end{array}$ & $\begin{array}{c}\text { Rectangular } \\
\text { Coil Design }\end{array}$ & $\begin{array}{c}\text { Double-Sided } \\
\text { Winding Coil }\end{array}$ \\
\hline Length of Copper wire Used Lcu (m) & 27.36 & 27.36 & 27.36 \\
\hline Cross-sectional Diameter of Coil (d) (mm) & 6 & 6 & 6 \\
\hline Airgap H (mm) & 200 & 200 & 200 \\
\hline X-Length of Coils Dlx (mm) & 600 & 600 & 450 \\
\hline Y-Length of Coils Dly (mm) & 600 & 600 & 450 \\
\hline Number of Coil Turn N & 18 & 18 & 30 \\
\hline Length of Ferrite Core (mm) & 60 & 60 & 45 \\
\hline Breadth of Ferrite Core (mm) & 60 & 60 & 45 \\
\hline Thickness of Ferrite Core (mm) & 6 & 6 & 6 \\
\hline Length of Aluminium Shield (mm) & 64 & 64 & 49 \\
\hline Breadth of Aluminium Shield (mm) & 64 & 64 & 49 \\
\hline Thickness of Aluminium Shield (mm) & 3 & 3 & 3 \\
\hline
\end{tabular}

\section{A. Ferrite Core Saturation Analysis of Resonant Coils}

The analysis is carried out with an airgap setting of $150 \mathrm{~mm}$. The ferrite core used has a magnetic saturation point of $0.43 \mathrm{~T}$. The result of the core saturation analysis and magnetic flux density distribution at a current of $50 \mathrm{~A}$ for the circular, rectangular and double-sided winding coils are shown in Figure 1. From the initial results, all the resonant coil design can withstand an electric current of $50 \mathrm{~A}$ without reaching the magnetic saturation limit of the ferrite core.

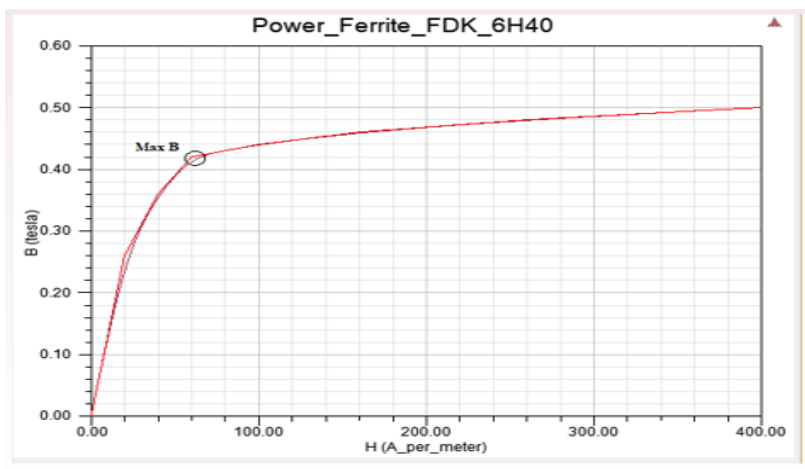

(a) The B-H Curve of Ferrite Material Used- FDK 6H40

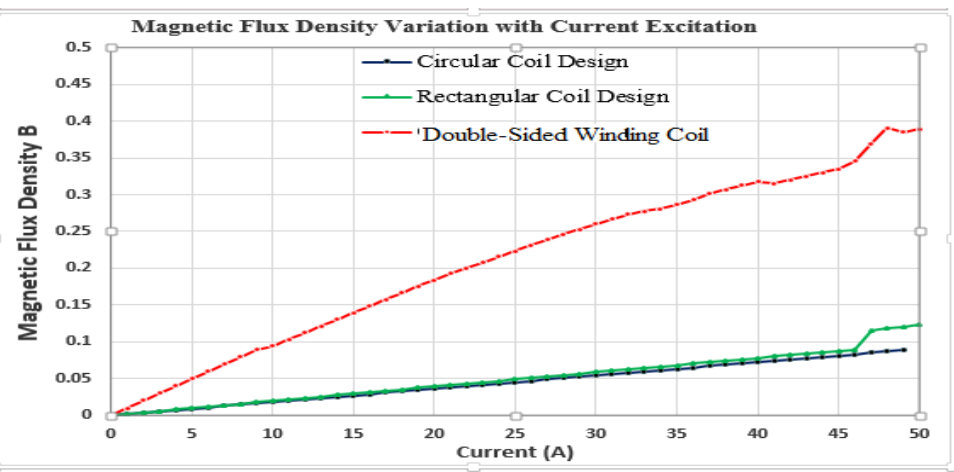

(b) Magnetic Flux Density Variation with Current Excitation

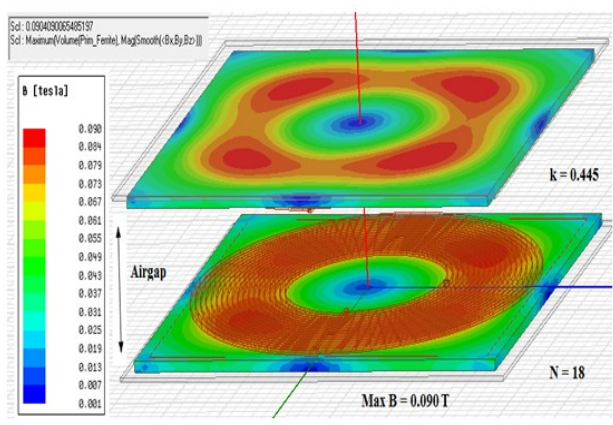

(c) Circular Resonant Coil

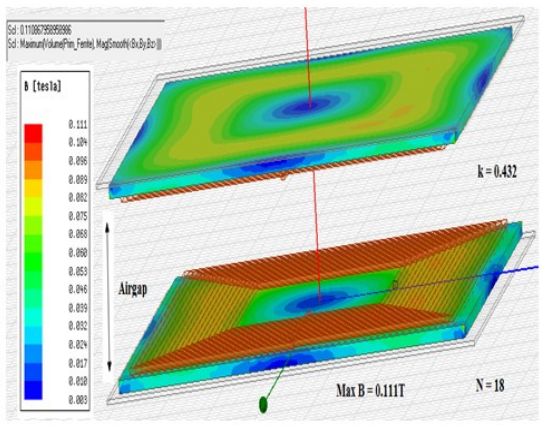

(d) Rectangular Resonant Coil

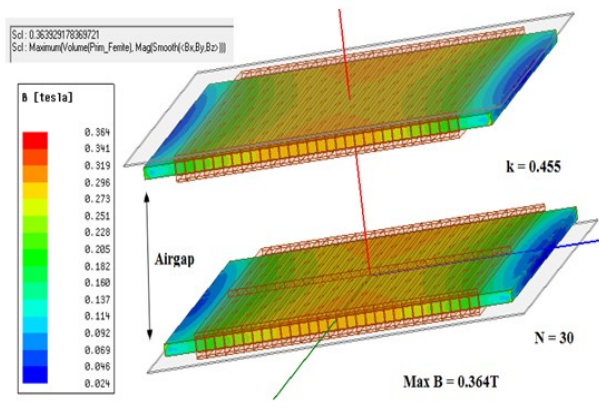

(e) Double-Sided Winding Resonant Coil

Fig. 1: Shapes, Coupling Factor k, and Magnetic Flux Density Distribution of Resonant Coil Designs 


\section{B. 3-D Parametric Analysis of Resonant Coils}

The parametric analysis was performed for lateral, longitudinal and vertical variation of the secondary coil. For the vertical airgap variation, the secondary coil position is varied along the $\mathrm{z}$-axis between $110 \mathrm{~mm}$ to $200 \mathrm{~mm}$ with step increase of 10 $\mathrm{mm}$. The lateral and longitudinal misalignment analysis is performed by varying the position of the secondary coil along the $\mathrm{x}$-axis and $\mathrm{y}$-axis respectively. Each of the secondary resonant coil designs were subjected to a lateral displacement

\section{FURTHER IMPROVEMENT AND OPTIMIZATION OF DOUBLE- SIDED WINDING COIL}

In the initial design of the double-sided winding coil, a coil parametric dimension size of $450 \mathrm{~mm}$ by length and $450 \mathrm{~mm}$ by breadth was used with 30 number of coil turns. This simple design gave a coupling coefficient of 0.33 at a $200 \mathrm{~mm}$ airgap, a coupling coefficient of 0.22 at a longitudinal misalignment of $300 \mathrm{~mm}$ and a coupling coefficient of 0.26 at a lateral displacement of $300 \mathrm{~mm}$ at a constant airgap of $200 \mathrm{~mm}$.

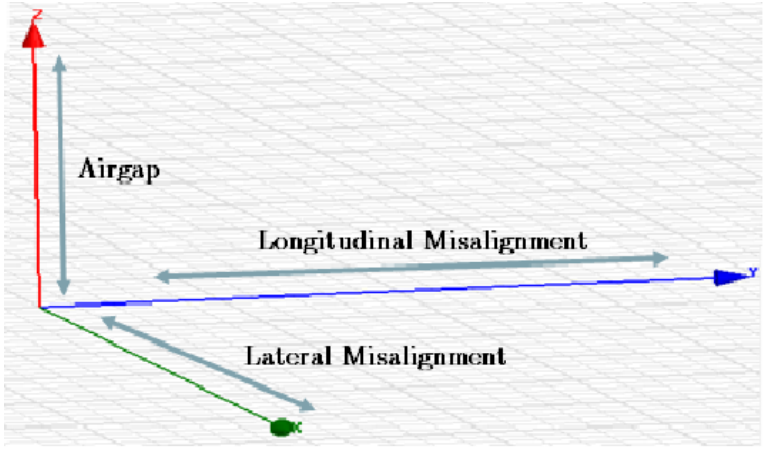

(a) The 3-D Orientation of Secondary coil.

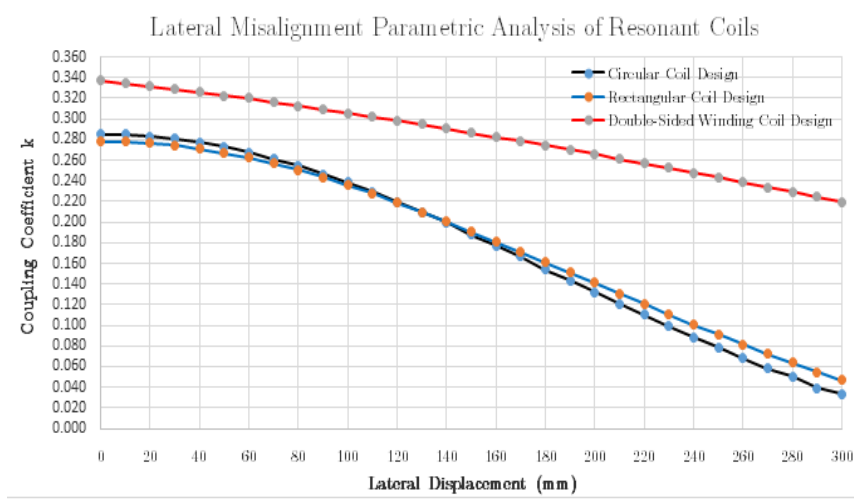

(c) Parametric Analysis of Lateral Misalignment of Coils

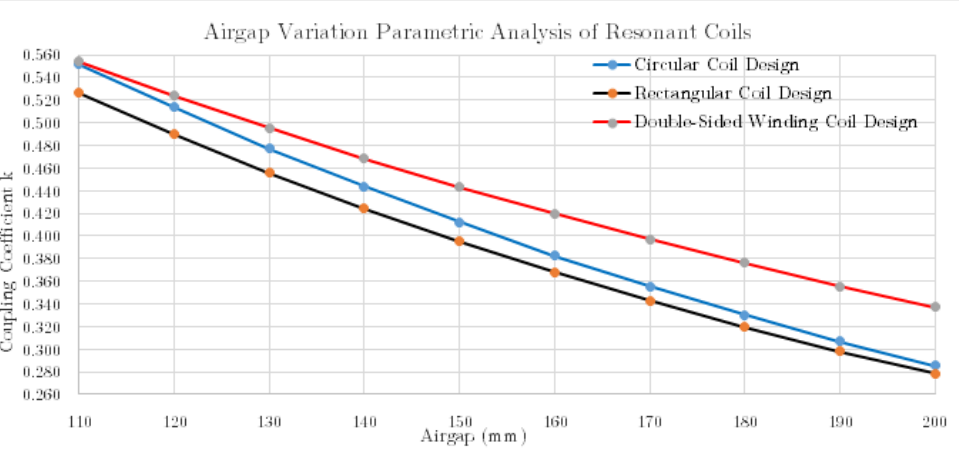

(b) Parametric Analysis of Airgap Variation of Resonant Coils

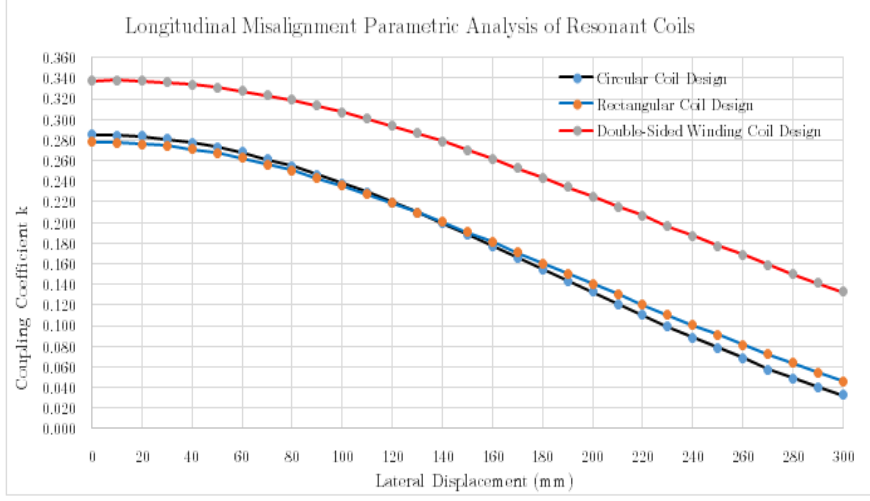

(d) Parametric Analysis of Longitudinal Misalignment of Coils

Fig. 2: Parametric Performance Analysis of Circular, Rectangular and Double-Sided Winding Resonant Coils

of between $0 \mathrm{~mm}$ and $300 \mathrm{~mm}$ with step increase of $10 \mathrm{~mm}$, while the longitudinal misalignment performance analysis was achieved by subjecting the secondary resonant coil to a longitudinal displacement of between 0 to $300 \mathrm{~mm}$ with a step increase of $10 \mathrm{~mm}$.

The coupling coefficient variation for each unit variation of the vertical, lateral and longitudinal displacement is shown in Figure 2. From the result shown in Figure 2, the circular and rectangular coils displayed the lower coupling coefficients at the maximum vertical, lateral and longitudinal displacements, as expected. On the other hand, the double-sided winding coil displayed better magnetic coupling performance at the maximum vertical, lateral and longitudinal displacements. The better performance of the double-sided winding coil is due to the reduction of the flux path to half the coil length, in addition to having a double-sided flux path.
A further improvement of the coil system can be achieved by altering the length and breadth of the coil with corresponding increase or decrease in the number of coil turns as determined by the requirement to use the same length of copper wire. This will alter the physical dimensions of the ferrite core and aluminum sheet shield, but the surface area and thickness of the ferrite core and aluminum sheet remains the same.

The first design alteration is to vary the length or breadth of the coil's physical size with a corresponding variation in the number of turns calculated in the process. Starting with an arbitrary step size of $50 \mathrm{~mm}$, and without exceeding the physical diameter of $600 \mathrm{~mm}$, four possible coil designs are generated and the physical modelling of the different coil designs produced. 


\section{A. Ferrite Core Saturation Analysis of Resonant Coils}

The analyses for the four additional designs were carried out at an airgap setting of $150 \mathrm{~mm}$ as this is the ground clearance for most electric cars. The ferrite core being used has a magnetic saturation point of $0.43 \mathrm{~T}$. The physical coil design parameters as well as the result of the core saturation analysis, magnetic flux density distribution, and maximum current for each of the coupling coefficient for each unit variation in the vertical, lateral and longitudinal displacement is shown in Figure 4.

From the results shown in Figure 4, can be seen that Model 3 and Model 4 coil designs offer better performance characteristics with respect to each unit of lateral displacement of the secondary coils when compared with the reference
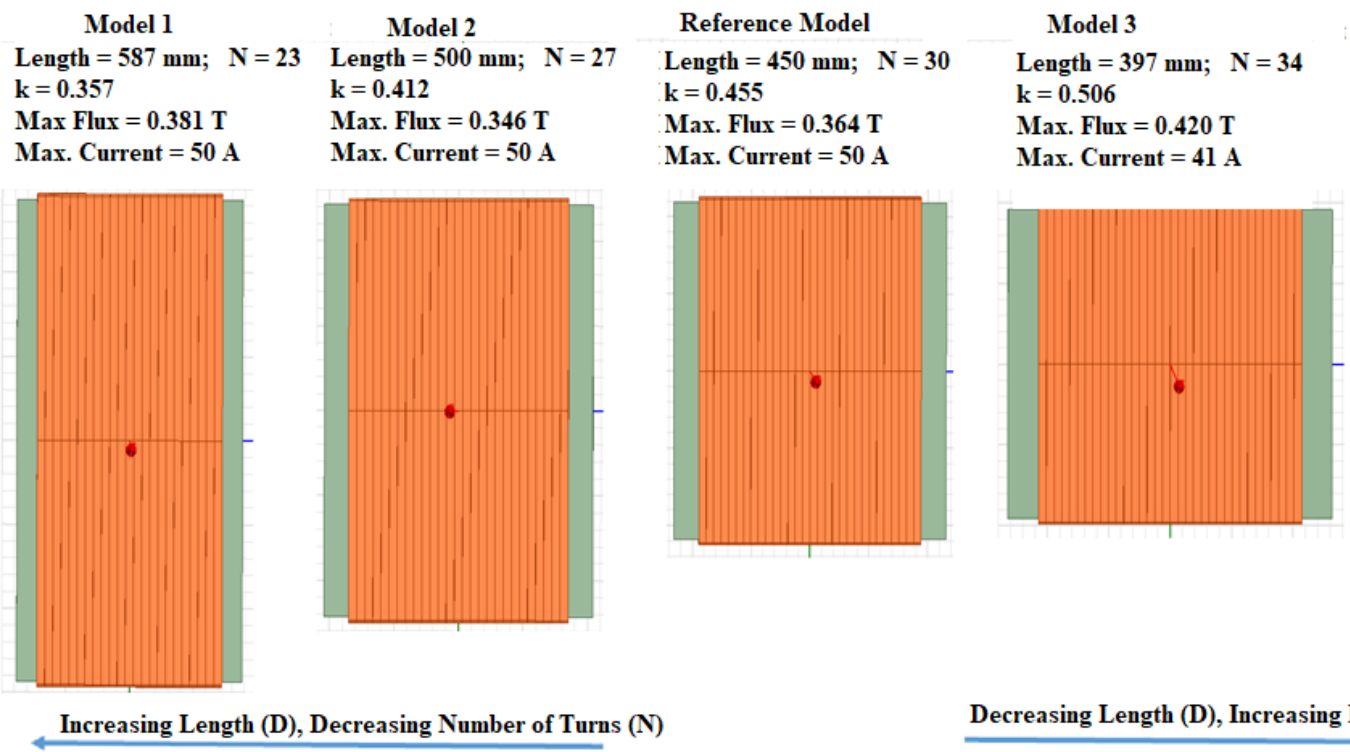

Model 4

Length $=355 \mathrm{~mm} ; \quad \mathrm{N}=38$ $\mathrm{k}=\mathbf{0 . 5 5 4}$

Max. Flux $=0.428 \mathrm{~T}$

Max. Current $=32 \mathrm{~A}$

Decreasing Length (D), Increasing Number of Turns (N)

Fig.3: Optimal Physical Design Variation and Maximum Flux Density Distribution of Double-Sided Winding Resonant Coil

four double-sided winding coil designs are shown at Figure 3. From the results in Figure 3, a reduction in the number of turns leads to a reduction in the coupling coefficient between coils. Also, increasing the coil length for the same number of turns reduces the inductance of the coil as well as the mutual coupling coefficient between coils. Thus, Model 1 and Model 2 are not optimum designs as the coupling coefficient values of 0.357 and 0.412 respectively for each of the design is less than that of the reference design of 0.445 , therefore these two designs are discarded. Model 3 with coupling coefficient of 0.455 and Model 4 with coupling coefficient of 0.506 offer a significant improvement above the reference design of 0.445 and further 3D parametric analysis was performed.

\section{A. 3-D Parametric Analysis of Optimized Double-Sided Winding Coil}

Parametric analysis was then performed for the lateral, longitudinal and airgap variation of the secondary coil.

For the vertical/airgap variation, the secondary coil is varied along the z-axis between $110 \mathrm{~mm}$ to $200 \mathrm{~mm}$ with step increase of $10 \mathrm{~mm}$. Also, each of the three secondary resonant coil designs (the reference model, model 3 and model 4) were subjected to a lateral displacement of between $0 \mathrm{~mm}$ to $300 \mathrm{~mm}$ with step increase of $10 \mathrm{~mm}$ while longitudinal misalignment performance analysis was done by subjecting the secondary resonant coil to a longitudinal displacement of between 0 to 300 $\mathrm{mm}$ with step increase of $10 \mathrm{~mm}$. The variation of magnetic model. Also model 4 offers better performance characteristics than Model 3; this is despite the fact that Model 3 has a longer coil length. This shows that the number of turns have a significant effect on the coupling coefficient rather than the length of the turns. Thus, given a constant length of copper wire wound round a rectangular ferrite core, the coupling factor between resonant coils is increases with increase in number of turns round the core.

Similarly, with respect to the variation of longitudinal displacement of the secondary coils, the lowest coupling coefficient of 0.18 and 0.24 was obtained at $300 \mathrm{~mm}$ longitudinal displacement while a strong coupling coefficient of close to 0.370 and 0.427 at no longitudinal misalignment respectively. This improved coupling result is expected due to the corresponding increase in the number of turns. The length of the coil turns having less effect in boosting the magnetic coupling.

The Model 3 show a coupling coefficient of 0.380 at $200 \mathrm{~mm}$ and a strong coupling coefficient of above 0.50 at an airgap setting of $140 \mathrm{~mm}$ while showing a strong coupling coefficient of close to 0.60 at the minimum airgap setting of $110 \mathrm{~mm}$. From the results in Figure 4, Model 4 shows a maximum strong coupling coefficient of 0.439 at the maximum airgap and coupling coefficient of 0.260 and 0.220 at the maximum lateral and longitudinal misalignment respectively. 


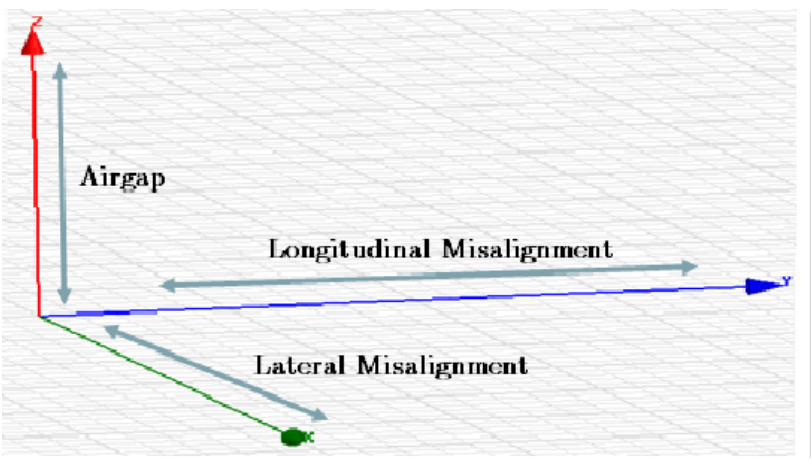

(a) 3-D Orientation of Optimized Secondary coil.

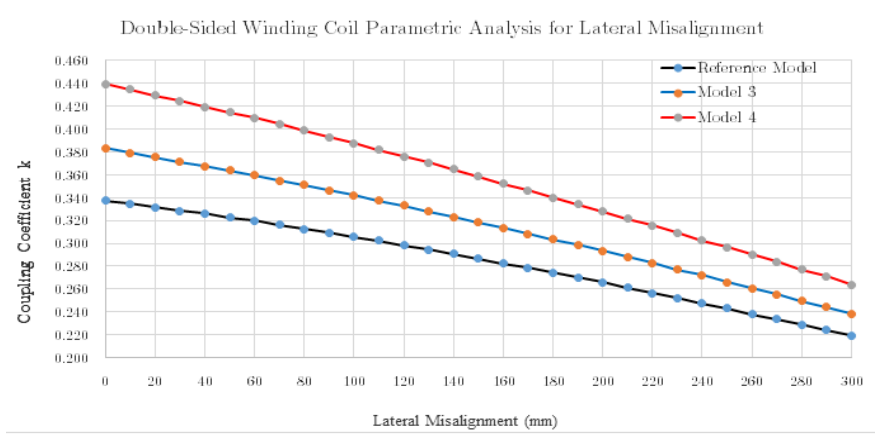

(c) Parametric Analysis of Lateral Misalignment of Coils

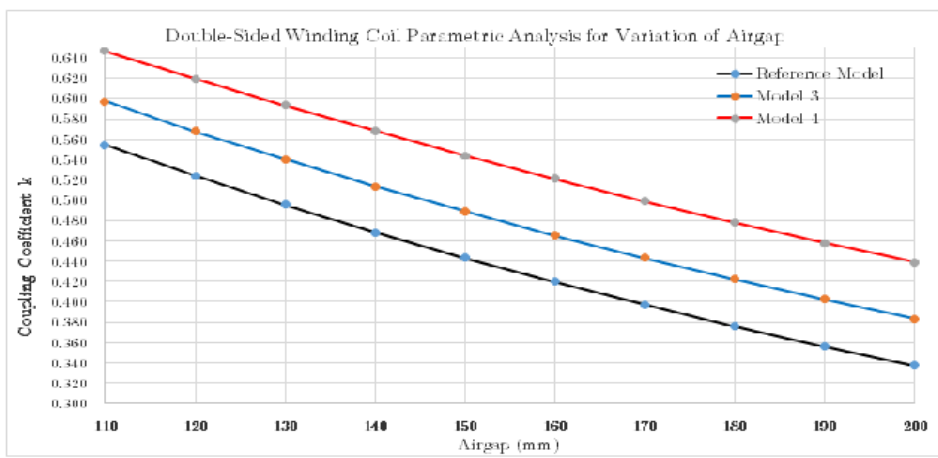

(b) Airgap Parametric Analysis of Optimized Resonant Coils

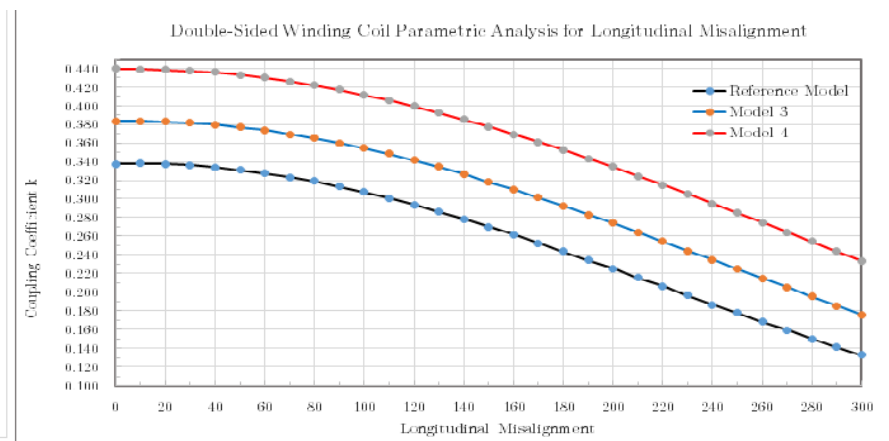

(d) Parametric Analysis of Longitudinal Misalignment of Coils

Fig. 4: Parametric Performance Analysis of Modified and Optimized Double-Sided Winding Resonant Coils.

Thus, achieving the threshold of acceptability as regards a viable design of resonant coils for bidirectional wireless power transfer for Electric Vehicles' use and application.

\section{CONCLUSION AND FUTURE WORKS}

This paper presents finite element modelling and 3-D parametric analysis of three simple resonant coil designs for bidirectional wireless power transfer for use with electric vehicle. The initial simulation results show that the doublesided winding coil offers the best magnetic coupling performance when compared with the circular and rectangular resonant coil designs. Further modifications and optimization of the double-sided winding coils produced two more highcoupled resonant coils which were further subjected to another round of finite element and 3-D parametric analysis.

From the finite element and 3-D parametric analysis, Model 4 with the maximum number of turn ratio offers the best performance in terms of mutual coupling coefficient for all cases analyzed. Future work will involve further physical modelling and parametric analysis of the optimized coil designs to ascertain the coil capability in terms of power transferred, optimum coil-to-coil efficiency and the optimal resonant frequency required for the transfer of optimum power.

\section{AKNOWLEDGEMENT}

This paper would like to acknowledge my research sponsor PTDF Nigeria and Mr. Konrad Yearwood for his contributions and technical advice in this research.

\section{REFERENCES}

[1] X. Mou and H. Sun, "Wireless Power Transfer: Survey and Roadmap," in 2015 IEEE 81st Vehicular Technology Conference (VTC Spring), 2015, pp. 1-5.

[2] O. Jonah, S. Member, S. V Georgakopoulos, and S. Member, "Wireless Power Transfer in Concrete via Strongly Coupled Magnetic Resonance," IEEE Trans. Antennas Propag., vol. 61, no. 3, pp. 1378-1384, 2013.

[3] D. Huang, Y. Urzhumov, D. R. Smith, K. Hoo Teo, and J. Zhang, "Magnetic superlens-enhanced inductive coupling for wireless power transfer," J. Appl. Phys., vol. 111, no. 6, 2012.

[4] K. A. Kalwar, M. Aamir, and S. Mekhilef, "Inductively coupled power transfer ( ICPT ) for electric vehicle charging - A review," Renew. Sustain. Energy Rev., vol. 47, pp. 462-475, 2015.

[5] G. Lempidis, "Wired and wire less charging of electri ic vehicles," pp. 1-7, 2014.

[6] K. Throngnumchai, A. Hanamura, Y. Naruse, and K. Takeda, "Design and Evaluation of a Wireless Power Transfer System with Road Embedded Transmitter Coils for Dynamic Charging of Electric Vehicles," pp. 1-10, 2013.

[7] S. Das, A. Wasif, N. Kumar, and E. Karim, "Wireless powering by magnetic resonant coupling: Recent trends in wireless power transfer system and its applications," Renew. Sustain. Energy Rev., vol. 51, pp. 1525-1552, 2015.

[8] D. Kurschner, C. Rathge, and U. Jumar, "Design methodology for high efficient inductive power transfer systems with high coil positioning flexibility," IEEE Trans. Ind. Electron., vol. 60, no. 1, pp. 372-381, 2013. 